\title{
Research on the Influence of Axisymmetric Endwall on EAT Performance
}

\author{
Han Teng ${ }^{1} \mathbb{D}$, Wanyang $\mathrm{Wu}^{2}$ and Jingjun Zhong ${ }^{2, * \mathbb{C}}$ \\ 1 Liaoning Province Key Laboratory of Marine Small Gas Turbine Technology, Marine Engineering College, \\ Dalian Maritime University, Dalian 116026, China; tenghangt@sina.com \\ 2 Merchant Marine College, Shanghai Maritime University, Shanghai 200120, China; wywu@shmtu.edu.cn \\ * Correspondence: zhongjj@shmtu.edu.cn; Tel.: +86-0411-8472-4665
}

check for

updates

Citation: Teng, H.; Wu, W.; Zhong, J. Research on the Influence of

Axisymmetric Endwall on EAT

Performance. Energies 2021, 14, 2215.

https://doi.org/10.3390/en14082215

Academic Editor: Alfredo Gimelli

Received: 18 February 2021

Accepted: 7 April 2021

Published: 15 April 2021

Publisher's Note: MDPI stays neutral with regard to jurisdictional claims in published maps and institutional affiliations.

Copyright: (c) 2021 by the authors. Licensee MDPI, Basel, Switzerland. This article is an open access article distributed under the terms and conditions of the Creative Commons Attribution (CC BY) license (https:/ / creativecommons.org/licenses/by/ $4.0 /)$.

\begin{abstract}
To improve the performance of electrically assisted turbochargers (EATs), the influences of the hub profile and the casing profile on EAT performance were numerically studied by controlling the upper and lower endwall profiles. An artificial neural network and a genetic algorithm were used to optimize the endwall profile, considering the total pressure ratio and the isentropic efficiency at the peak efficiency point. Different performances of the prototype EAT and the optimized EAT under variable clearance sizes were discussed. The endwall profile affects an EAT by making the main flow structure in the endwall area decelerate and then accelerate due to the expansion and contraction of the meridional surface, which weakens the secondary leakage flow of the prototype EAT and changes the momentum ratio of the clearance leakage flow and the separation flow in the suction surface corner area. Because the tip region flow has a more significant influence on EAT performance, the optimal casing scheme has a better effect than the hub scheme. The optimization design can increase the isentropic efficiency of the maximum efficiency point by $1.5 \%$, the total pressure ratio by $0.67 \%$, the mass flow rate by $1.2 \%$, and the general margin by $6.4 \%$.
\end{abstract}

Keywords: axisymmetric endwall; electrically assisted turbocharger; axial compressor; optimization design

\section{Introduction}

As a new type of supercharger solution, the electrically assisted turbocharger (EAT) has shown potential in reducing engine emissions and improving maneuverability [1]. In the field of vehicle power (with the popularity of mild hybrid and moderate hybrid vehicles), the 48-V onboard power system provides the basis for the large-scale installation of EATs [2]. In the field of fuel cells, the EAT is an important component for the miniaturization of fuel cells [3]. In the marine power field, an EAT can be used as an alternative to the auxiliary blower to improve the efficiency of the power plant and reduce the load on the power grid. At present, EATs mostly use the radial compressor impeller of the existing turbochargers. Consequently, the research team proposed a design idea of an axial compressor for the EAT rotor, aiming at applications in large vehicle power plants and small ship power plants; that is to say, systems with a high mass flow rate, a low-pressure ratio, and direct connections with high-speed motors [4].

In the early design of compressors, linear and uniformly contracted endwall were often used, such as the uniform-diameter design. However, with the accumulation of design experience, researchers have gradually discovered that a linear endwall is not optimal and appropriate axisymmetric endwall modeling significantly affects the compressor performance by influencing the endwall flow structure. Hoeger [5] studied the influence of the endwall shape on a shock wave system in a transonic plane cascade and tested a linear hub and concave hub in a high-speed wind tunnel. He found that the original oblique shock wave of the concave hub became a positive shock wave, which demonstrates that a change in endwall shape can considerably affect the flow near the endwall and the flow 
field structure of the span direction. Georg [6,7] thoroughly studied the influence of an axisymmetric casing on the performance of the second-stage rotor blades of the subsonic high-pressure compressor of the Siemens AG and optimized the casing profile of the secondstage rotor blades. After the transfer rules were obtained, the last six stages of the entire compressor stage were optimized, and the entire stage compressor was simulated, the results of which show that the optimized compressor can improve the isentropic efficiency of the design point by $0.3 \%$. Xu [8] studied the effects of concave, convex, and wavy hub profiles on the performance of transonic compressor rotors and found that the hub profile could affect the shock position and radial flow of the compressor rotors. Although the concave hub can improve the rotor performance, considering the balance between load and efficiency, the wavy hub was considered a more appropriate choice. Yang [9] investigated how concave hubs affect the separation of the stator blades in transonic compressors and discovered that modifying the hub can effectively improve corner blockage and restrain separation. The optimal modification can increase the total pressure recovery coefficient from 0.89 to 0.948 at a height of $10 \%$ of the stator blade. Zhang [10] examined the influence of concave axisymmetric endwall on the performance of high-load compressor cascades with large installation angles by extracting and extending the blade profiles into a plane cascade and discussed the effects of the concave coverage depth and coverage distance in terms of eliminating the separation of the suction surface using the plane cascade. Then, a concave casing was applied to the stator blades of the last 1.5 stage compressor on a ground gas turbine, improving the peak efficiency by $0.94 \%$. Sun [11] studied the influences of two types of hub profiles (concave-convex and concave-convex) on the performance of a low-reaction-degree, highly loaded adsorption transonic compressor rotor and found that the hub profile affects the intensity of both the blade tip leading edge shock wave and the blade root outlet shock wave. The convex-concave hub profile increased the flow capacity but decreased the peak pressure ratio and peak efficiency; in contrast, the concave-convex profile reduced the flow capacity but increased the peak pressure ratio and peak efficiency. Liu [12] analyzed the inhibitory effect of a concave hub on the backflow of the suction surface in a large bypass ratio fan and revealed that the concave hub's influence on the flow at the root of the fan is achieved mainly by the increase in streamline curvature due to the concave hub, thereby achieving a flow acceleration. The concave hub was further reported to have a monotonic improvement in both the pressure ratio and the efficiency with an increasing concave depth, and a concave shape closer to the separation location was found to yield better results.

To date, many scholars and research institutions have studied the influence of the endwall profile on compressor performance, focusing mostly on transonic compressors and plane cascades. However, an EAT rotor works under subsonic conditions, and thus, the tangential velocities of the hub and casing positions differ greatly. Moreover, the flow radial migration phenomenon caused by the centrifugal force and the Coriolis force is obvious. To improve the performance of the EAT rotor at the design point, the influences of the hub profile and the casing profile on the EAT rotor were explored in this paper. In addition, the EAT endwall profile was optimized by using an artificial neural network and a genetic algorithm. The performance differences between the prototype and the optimized EAT under different height clearances, and the reasons for these differences, were studied.

\section{Research Object and Numerical Method}

\subsection{Research Object}

The research object of this paper is a self-designed prototype EAT rotor. To maximize the total pressure ratio of the EAT, a design with an equal outer diameter was adopted. Table 1 shows the performance and geometric parameters of the prototype compressor at the design point. Since there is no restriction on matching with the rear row of the vane, to ensure efficiency, all bending angles and aerodynamic loads at the root, middle and tip of the blade exceed the design limits of conventional compressors (load factor $>0.6$ ). Figure 1 is the Smith chart which presented the ranges of the flow coefficient and load coefficient 
of traditional and high-load design compressors based on the tangential velocity at the middle diameter of the blade. The load coefficient and flow coefficient of the prototype EAT are clearly far from the high-load design area, but the prototype can maintain a high isentropic efficiency. Figure 2 shows a 3D geometric model of the prototype EAT.

Table 1. Geometric parameters and design point performance of the self-designed electrically assisted turbochargers (EAT) rotor.

\begin{tabular}{cccc}
\hline Parameter & Value & Parameter & Value \\
\hline External diameter $(\mathrm{mm})$ & 83 & Mean diameter solidity & 1.64 \\
Inlet hub ratio & 0.45 & Mass flow $(\mathrm{kg} / \mathrm{s})$ & 0.629 \\
Rotation speed $(\mathrm{rpm})$ & 60,000 & Pressure ratio & 1.346 \\
Mean diameter aspect ratio & 0.68 & Isentropic efficiency & $88.68 \%$ \\
\hline
\end{tabular}

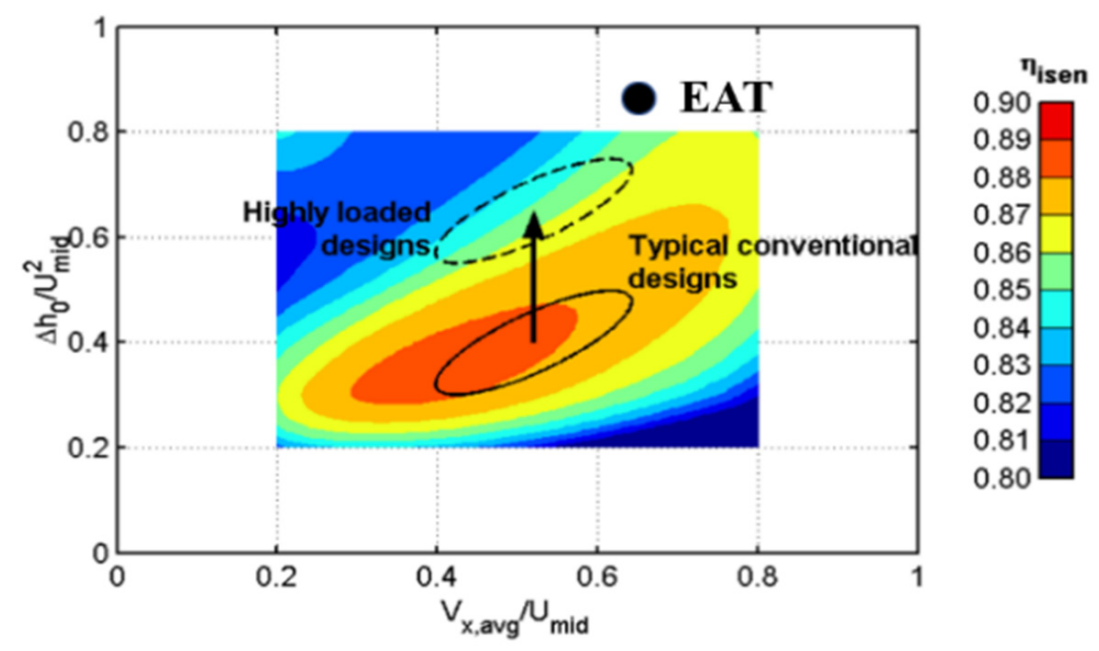

Figure 1. Smith chart.

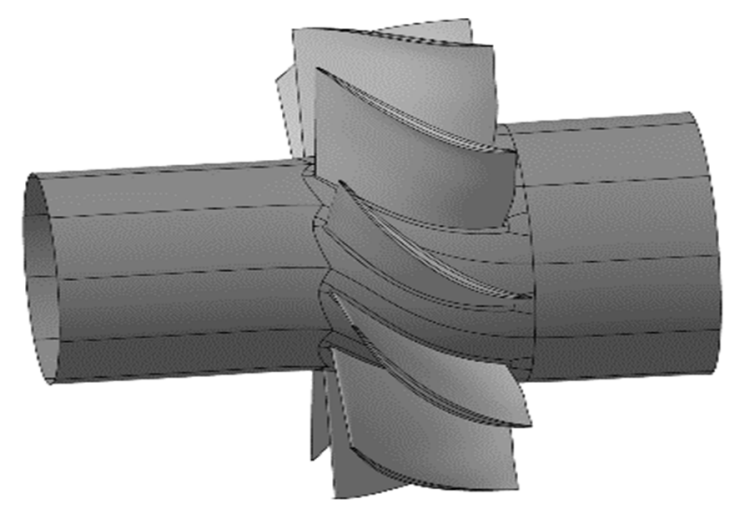

Figure 2. 3D model of the prototype EAT.

\subsection{Discrete Modification Scheme}

A summary of the published studies reveals that a concave axisymmetric endwall can significantly improve the performance of compressor rotors. Therefore, a concave axisymmetric endwall was selected as the research object in this paper. Specifically, the hub profile and the casing profile in the prototype blade area were evenly divided into 10 parts according to the axial chord length, and the leading and trailing edge points were fixed. First, the influence of the concave position on the EAT performance was studied. The performance peak was found while modifying the axial position as the variable and the concave depth was varied: the depth of the concave shape was spaced at $3 \%$ blade height 
(h, height), and all modification profiles were smoothly connected by B-spline curves to form different endwall shapes. To control the other variables, when modeling one axial position, the remaining variables which control the other position were set at fixed values. Figure 3 presents a schematic diagram of the endwall modification scheme and the specific modeling schemes of the concave endwall are described in Table 2.

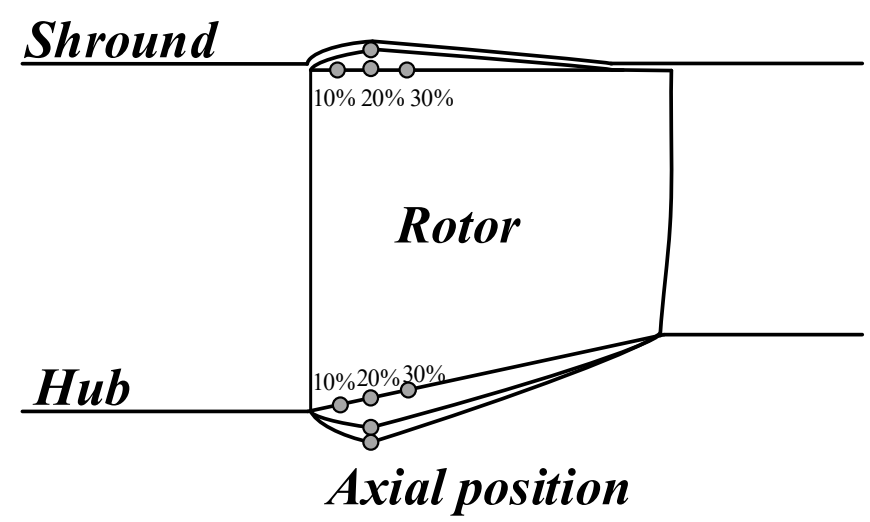

Figure 3. Schematic diagram of the endwall modification scheme.

Table 2. Specific modeling schemes of the concave endwall.

\begin{tabular}{cccccc}
\hline $\begin{array}{c}\text { Hub } \\
\text { Modification }\end{array}$ & $\begin{array}{c}\text { Axial } \\
\text { Position }\end{array}$ & $\begin{array}{c}\text { Concave } \\
\text { Depth }\end{array}$ & $\begin{array}{c}\text { Casing } \\
\text { Modification }\end{array}$ & $\begin{array}{c}\text { Axial } \\
\text { Position }\end{array}$ & $\begin{array}{c}\text { Concave } \\
\text { Depth }\end{array}$ \\
\hline Hub-10\%-0.06 h & $10 \%$ & $0.06 \mathrm{~h}$ & Shr-10\%-0.03 h & $10 \%$ & $0.03 \mathrm{~h}$ \\
Hub-20\%-0.06 h & $20 \%$ & $0.06 \mathrm{~h}$ & Shr-20\%-0.03 h & $20 \%$ & $0.03 \mathrm{~h}$ \\
Hub-20\%-0.09 h & $20 \%$ & $0.09 \mathrm{~h}$ & Shr-20\%-0.06 h & $20 \%$ & $0.06 \mathrm{~h}$ \\
Hub-20\%-0.12 h & $20 \%$ & $0.12 \mathrm{~h}$ & Shr-20\%-0.09 h & $20 \%$ & $0.09 \mathrm{~h}$ \\
Hub-20\%-0.15 h & $20 \%$ & $0.15 \mathrm{~h}$ & Shr-30\%-0.03 h & $30 \%$ & $0.03 \mathrm{~h}$ \\
Hub-30\%-0.06 h & $30 \%$ & $0.06 \mathrm{~h}$ & Shr-40\%-0.03 h & $40 \%$ & $0.03 \mathrm{~h}$ \\
\hline
\end{tabular}

\subsection{Numerical Method}

For the numerical simulation, a Numeca/Autogrid5 software was employed for meshing, and the solver was Numeca/FINE. The simulation type was a 3D steady simulation. The grid height of the first layer was $0.34 \mu \mathrm{m}$, and the $\mathrm{Y}+$ value of the first layer was approximately 1 . The inlet was set to a total pressure inlet of $101,325 \mathrm{~Pa}$, the outlet was set to a middle-diameter static pressure of $108,500 \mathrm{~Pa}$, and the other positions were assigned by a radial equilibrium equation. For both lateral sides in the computation zone, the periodic boundary condition was applied. The walls were set as smooth, adiabatic, and nonslip walls. To ensure the grid independence of the numerical solution, four sets of grids with total grid numbers of $0.6 \times 10^{6}, 0.9 \times 10^{6}, 1.2 \times 10^{6}$, and $1.5 \times 10^{6}$ were drawn (named coarse, medium1, medium2, and fine, respectively). The characteristic lines at the design speed for these four grids are plotted in Figure 4, which reveals that when the number of grids reaches $1.2 \times 106$, the performance parameters and flow field details are independent of the number of grids. Therefore, $1.2 \times 10^{6}$ grids are employed in this paper for further research. 


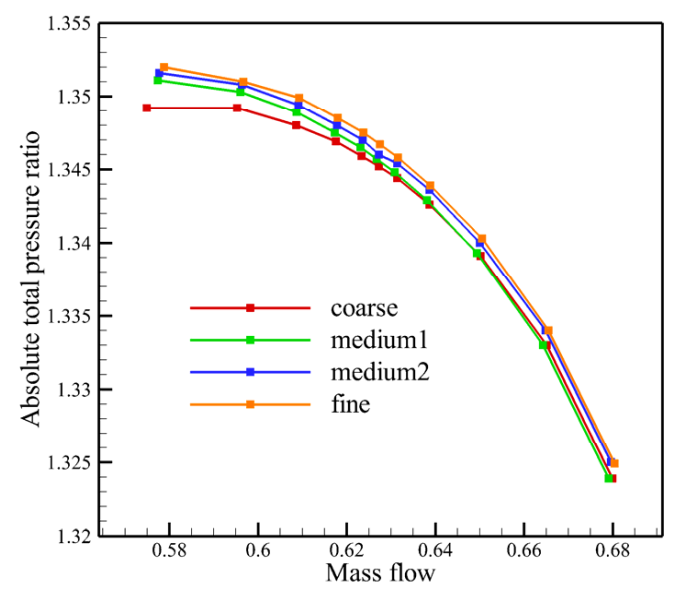

Figure 4. Grid independence research.

To validate the numerical simulation results, there was no relevant test result, since the EAT is still in the aerodynamic design stage. Hence, the Spalart-Allmaras (S-A) turbulence model was used in this simulation, which originated from an airfoil field simulation of an outflow field and has strong robustness to complex flow fields with an adverse pressure gradient. As a compromise between the 0-equation Baldwin-Lomax model and the 2equation $\mathrm{k}-\varepsilon$ model, the S-A model can reduce the time consumed by many numerical simulations due to automatic optimization and accurately simulate the details of the flow field. The turbulence models for the transonic compressor rotor studied by Zhang [9] and the subsonic rotor studied by Gao [13] are both low Reynolds number S-A models, whose characteristic Reynolds number ranges include the Reynolds number of the EAT rotor. Thus, for the EAT rotor, the S-A model was deemed capable of simulating the actual situation with relative accuracy for the separation caused by tip leakage flow. Figure 5 depicts the prototype EAT grid division and the design flow point boundary condition settings.

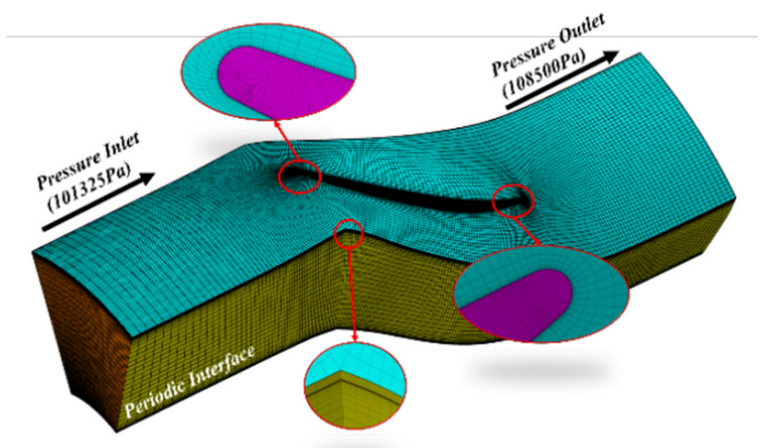

Figure 5. Prototype EAT grid.

\section{Analysis of the Results}

\subsection{Characteristic Line Comparison}

The characteristic lines of the normalized mass flow-total pressure ratio and normalized mass flow-isentropic efficiency after the axisymmetric concave modification of the hub and casing are plotted in Figure 6a,b, respectively. An analysis of the results reveals that, at $3 \%$ concavity, the hub part had no obvious effect on the rotor performance, so the relevant characteristic lines were not drawn. For the hub modification, with an increase in concave axial position, the pressure ratio and efficiency first increased and subsequently decreased. The optimal concave position was at $20 \%$ of the axial chord length. With increasing concave depth, both pressure ratio and efficiency increased first and subsequently decreased, and the optimal concave depth was at $12 \%$ of $h$. The casing modification exhibited the same 
variation trend as the hub modification; the optimal concave position was at $20 \%$ of the axial chord strength, and the optimal concave depth was at $6 \%$ of $h$.

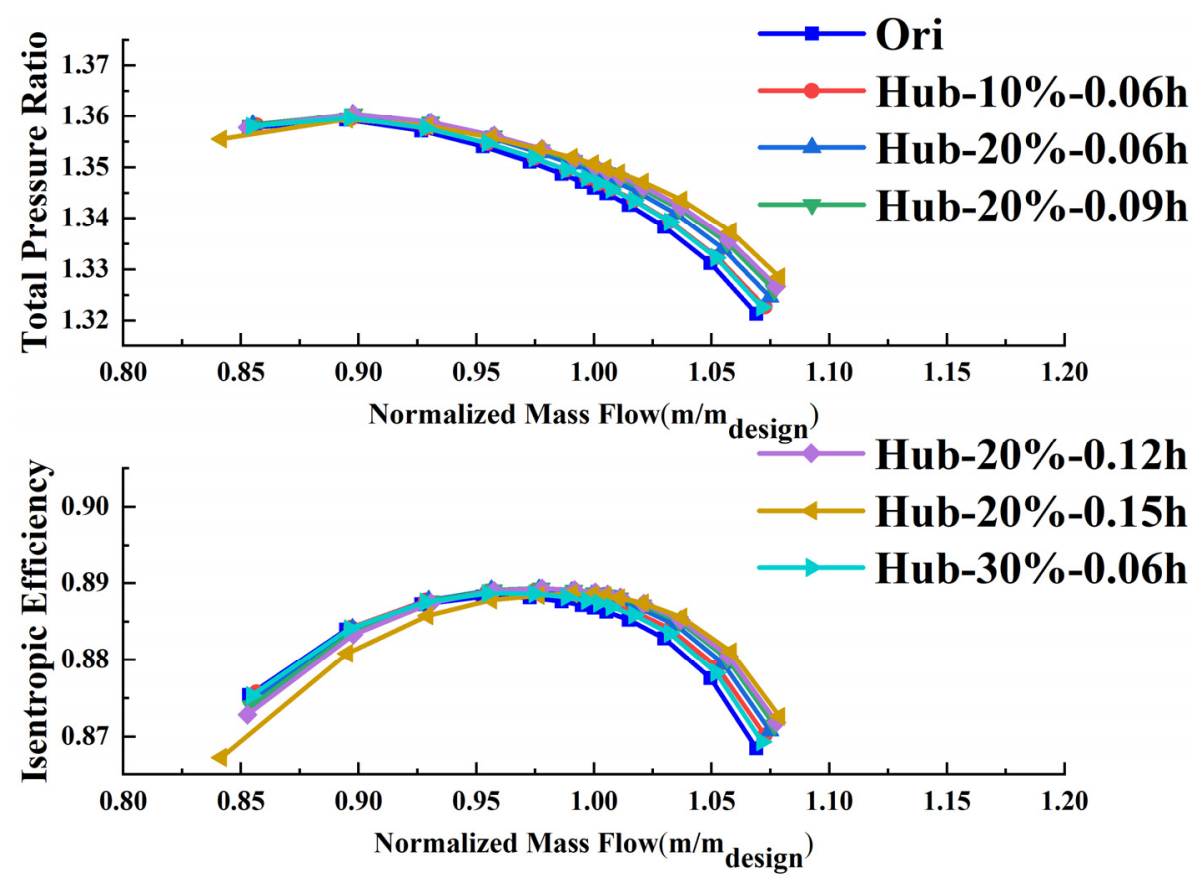

(a)
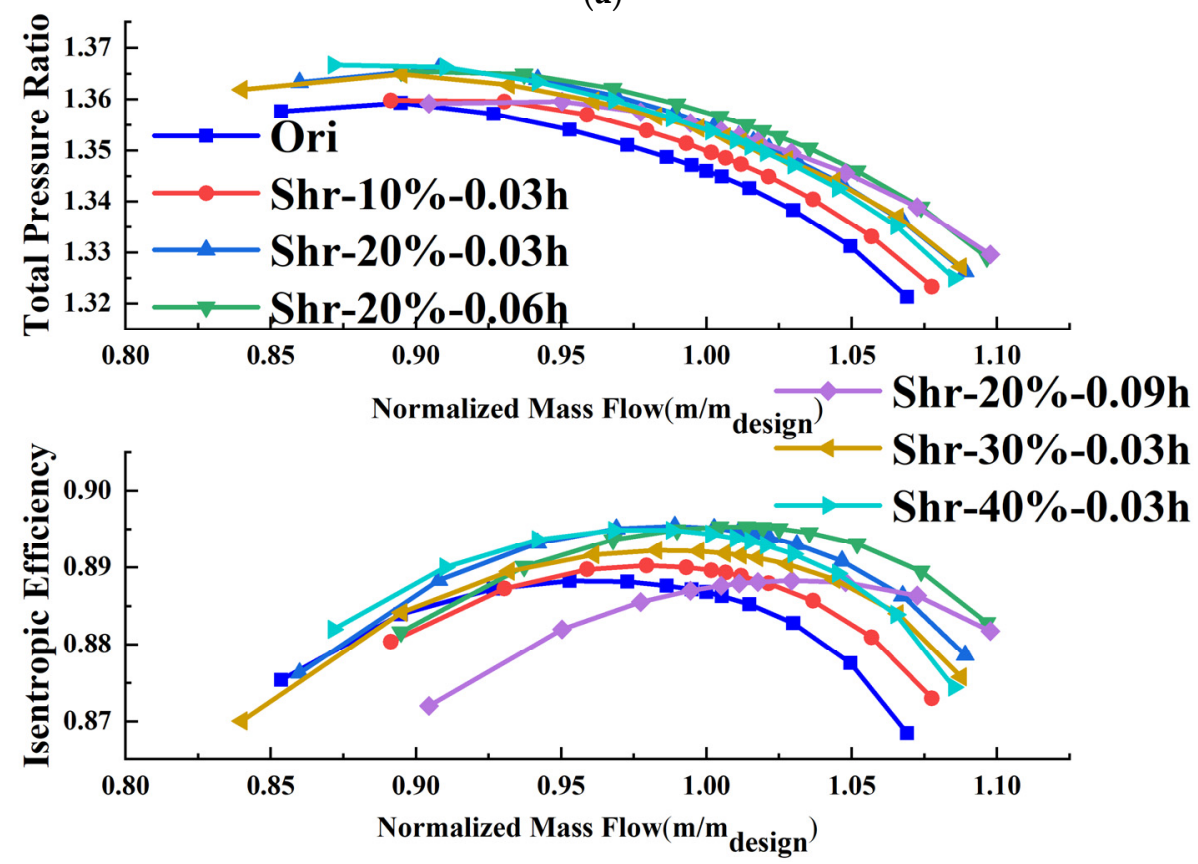

(b)

Figure 6. (a) Hub modification characteristic lines; (b) casing modification characteristic lines.

According to the characteristic line comparison, the hub concave profile can weakly improve the EAT performance, while the casing concave profile can significantly improve it. This discrepancy is due to the high EAT rotating speed $(60,000 \mathrm{rpm})$ and the moderate hub ratio. Hence, the tangential velocity of the blade increases with the blade height, which makes the absolute flow capacities at the blade root and blade tip greatly differ. Moreover, under the effect of centrifugal force, the flow from the leading edge of the hub into the flow passage starts to rise from the mid-chord position to the tip position, which intensifies the flow nonuniformity in the spanwise direction. The definition of the 
throughflow coefficient is shown in Equation (1), where $\rho$ is the density, $V_{Z}$ is the axial velocity, and $U$ is the blade tangential velocity. Figure 7 shows the distribution of the outlet mass-averaged throughflow coefficient along the blade height at the peak efficiency point. Table 3 describes the surface integral results of the mass-averaged throughflow coefficient in the blade root area (0-30\% blade height), the middle area (30-80\% blade height) and the tip area (80-100\% blade height).

$$
\phi=\rho V_{Z} / U
$$

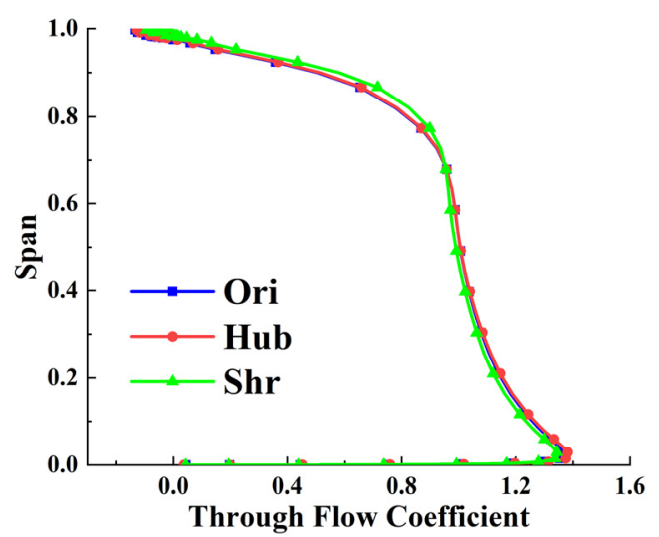

Figure 7. Through flow coefficient distribution.

Table 3. Surface integral results of mass-averaged throughflow coefficient.

\begin{tabular}{cccc}
\hline & Root & Midspan & Tip \\
\hline Prototype & 1.165 & 0.984 & 0.587 \\
Optimal Hub & 1.171 & 0.985 & 0.591 \\
Optimal casing & 1.151 & 0.984 & 0.621 \\
\hline
\end{tabular}

The throughflow coefficient represents the throughflow capacity at a given tangential velocity. As shown in Figure 7, at the peak efficiency point, the concave hub enhances the flow capacity below the $15 \%$ span, while the flow capacity above the $15 \%$ span remains basically unchanged. The flow capacity of the concave casing below $65 \%$ is smaller than that of the prototype, whereas the flow capacity of the span of $65-100 \%$ is larger than that of the prototype. Table 3 shows that the concave hub improves the flow coefficient at the root by $0.5 \%$ and that at the tip by $0.7 \%$; in contrast, the concave casing reduces the flow coefficient at the root by $1.2 \%$ and increases the flow coefficient at the tip by $5.7 \%$. From the definition of the throughflow coefficient, on the premise of a constant coefficient value, a higher tangential velocity has a higher absolute flow capacity. This means that the concave casing has a more obvious influence on the EAT performance than does the concave hub. Therefore, in the following analysis, after briefly analyzing the reasons for the performance improvement of the concave hub, the main reasons for the performance improvement of the concave casing will be analyzed in detail.

\subsection{Flow Detail Analysis}

Figure 8 shows a comparison between the distributions of the static pressure coefficient (Cp) and the isentropic Mach number (iMa) on the 5\% span chordwise blade surface of the prototype EAT and the optimal concave hub EAT at the peak efficiency point. $C p$ is defined in Equation (2), wherein $P_{r t p}$ is the relative total pressure and $P_{a}$ is the atmospheric pressure. 


$$
C_{p}=\left(P_{r t p}-P_{a}\right) /\left(0.5 *\left(\rho U^{2}\right)\right)
$$
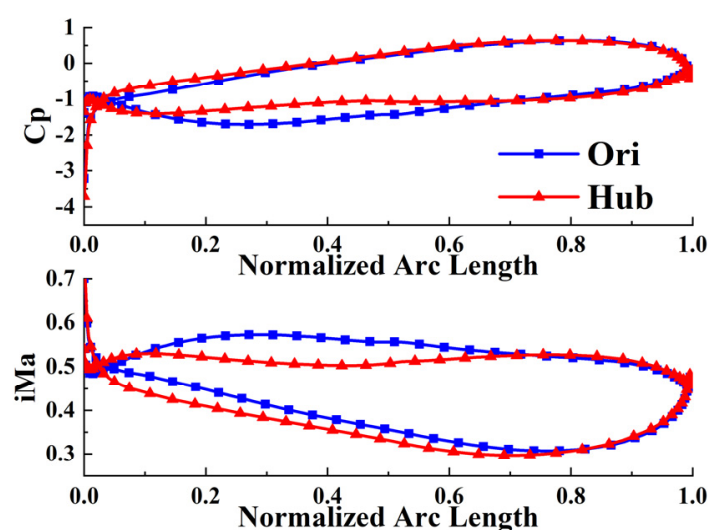

Figure 8. Chordwise distributions of the static pressure coefficient (Cp) and the isentropic Mach number (iMa) at $5 \%$ of the span of the prototype and the optimal concave hub.

Figure 8 demonstrates that the concave hub increases the static pressure on the suction surface at $10-60 \%$ of the chord length, while the static pressure on the pressure surface basically remains unchanged. Thus, the pressure difference between the suction and the pressure surface is reduced, which reduces the front chord aerodynamic load and improves the flow state. According to the iMa distribution, from $20 \%$ of the chord length, the flow velocities on the suction surface and the pressure surface are lower than those of the prototype, but these velocities are consistent with those of the prototype at $80 \%$ of the chord length. The reason for this is that the curvature change of the concave hub makes the meridian channel first exhibit a local expansion and then appeared a contraction shape on the basis of overall contraction. The flow in the concave area exhibits a decelerationacceleration phenomenon, which weakens the leading edge suction peak of the prototype, reduces the blade profile loss, and improves the flow efficiency.

Figure 9 shows a comparison between the distributions of $\mathrm{Cp}$ and iMa on the $95 \%$ span chordwise blade surface of the prototype EAT and the optimal concave casing EAT at the peak efficiency point. From this figure, as a result of the concave casing, the Cp on the suction surface is lower than that of the prototype at $25 \%$ of the chord length; in the following flows, the $\mathrm{Cp}$ is higher than that of the prototype, reflecting a front-loaded characteristic. Beyond 70\% of the chord length, the $\mathrm{Cp}$ is identical to that of the prototype. Regarding the iMa distribution, the change in the meridian plane shape in the tip area affects the element blade profile flow and the clearance leakage flow near the casing, so the iMa at $95 \%$ of the span reaches the maximum value in advance at $20 \%$ of the axial chord length, which is higher than that of the prototype. In contrast, the iMa is smaller than that of the prototype from $25 \%$ to $70 \%$ of the axial chord length, and from $70 \%$ of the chord length to the trailing edge, the iMa is restored to the value of the prototype. Compared with the prototype EAT, the implementation of a concave casing makes the original once "acceleration-deceleration" trend on the suction surface become twice, the Mach number on the blade surface to increase more smoothly, and the efficiency in the tip area to improve. 

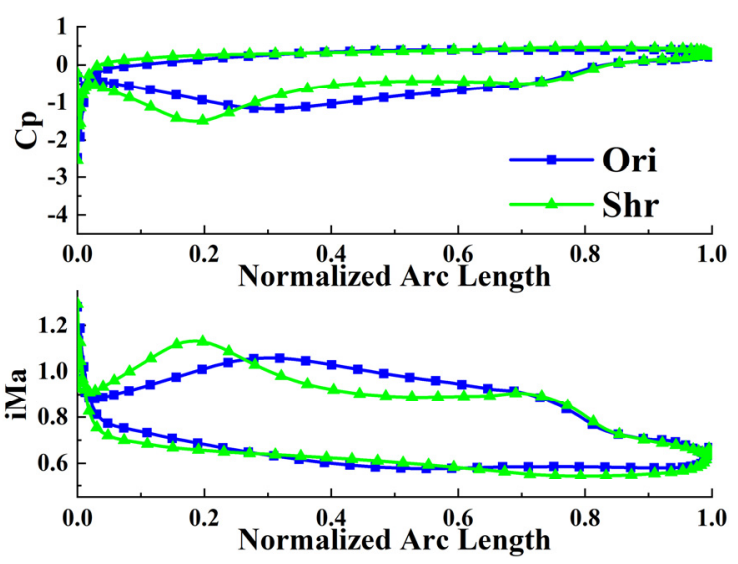

Figure 9. The chordwise distributions of $\mathrm{Cp}$ and iMa at $95 \%$ of the span of the prototype and the optimal concave casing.

Figure 10a,b show the outlet entropy contours of the prototype and the optimal concave casing, respectively, at the peak efficiency point. The entropy of the prototype outlet section is $8.06 \mathrm{~J} /\left(\mathrm{Kg}^{*} \mathrm{~K}\right)$, while the mass-averaged entropy of the concave casing outlet section is $7.50 \mathrm{~J} /\left(\mathrm{Kg}^{*} \mathrm{~K}\right)$. From these contours, the large-scale loss caused by tip leakage is inhibited in the concave casing, which results in decreased entropy. Near the casing, a small number of high-entropy zones that are not found in the prototype appear because of a separation flow in the suction surface corner caused by the contraction of the casing. The concave casing limits the high-entropy region to the tip. Although a local region with a higher entropy than the prototype appears near the casing, the overall influence range of the high-entropy region at the tip is smaller than that of the prototype. Figure $11 a, b$ show the static pressure distributions at $99 \%$ of the span height on the S1 surface of the prototype EAT and the optimal concave casing EAT, respectively, at the peak efficiency point. The static pressure chute in the tip area represents the direction of the clearance leakage vortex [14]. The static pressure distribution shows that the concave casing slows down the flow at the leading edge, enlarges the angle between the tip leakage vortex and the chord direction of the blade, and expands the low static pressure area inside the whole tip passage. As shown in Figure 11a, the middle chord of the blade has an area with a large pressure gradient and a small static pressure chute appears, which corresponds to the secondary leakage flow. In the optimal concave EAT, this secondary leakage phenomenon is suppressed because the contraction section of the concave casing enhances the velocity of the clearance leakage vortex to maintain the original direction of the movement.

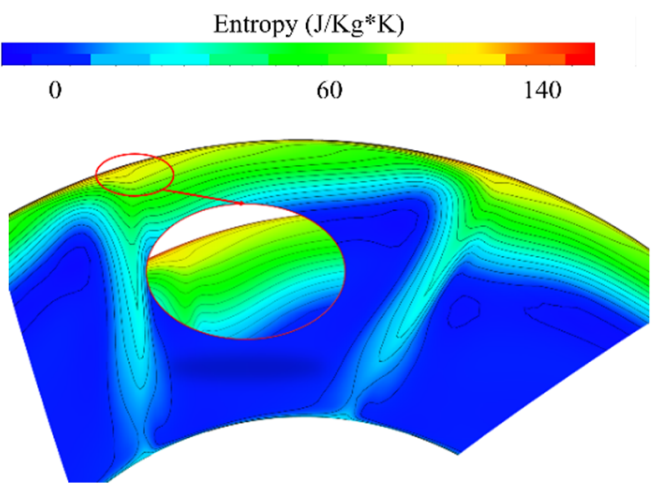

(a) prototype

Figure 10. Cont. 


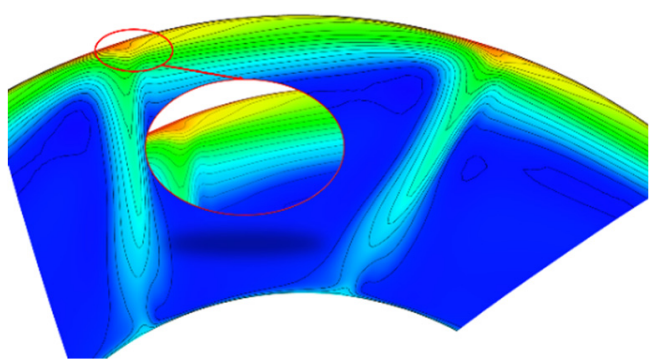

(b) optimal concave casing

Figure 10. Outlet entropy contours.

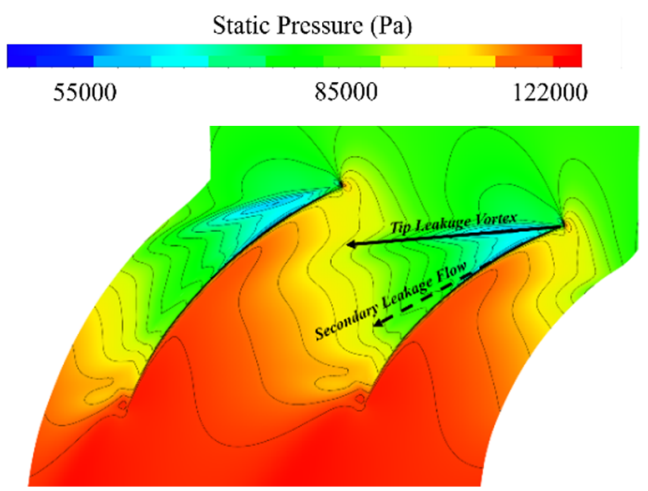

(a) prototype

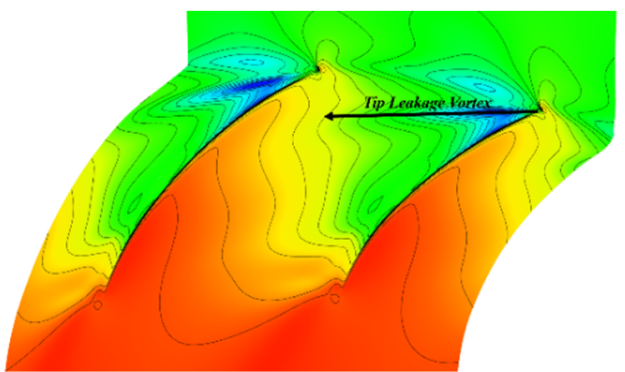

(b) optimal concave casing

Figure 11. The S1 surface static pressure contour at $99 \%$ of the span.

Figure 12 shows the entropy distribution at $99 \%$ of the span on the S1 surface of the prototype EAT and the optimal concave casing EAT at the peak efficiency point. In the prototype, there is a strong entropy increase at the middle and rear chord of the tip. This high-entropy zone across the entire channel is due to the excessive load of the prototype. Due to this excessive load, not all of the leakage fluid flows out of the current channel; instead, some of the fluid crosses the pressure surface of the adjacent blades, resulting in secondary leakage. In contrast, the deceleration-acceleration trend caused by the concave casing allows most of the clearance leakage fluid to flow out of the current channel; although the front-slowing expansion causes tip clearance leakage flow, the highentropy area expands, but the whole concave casing still shows an inhibitory entropy effect, improving the whole tip flow efficiency. 


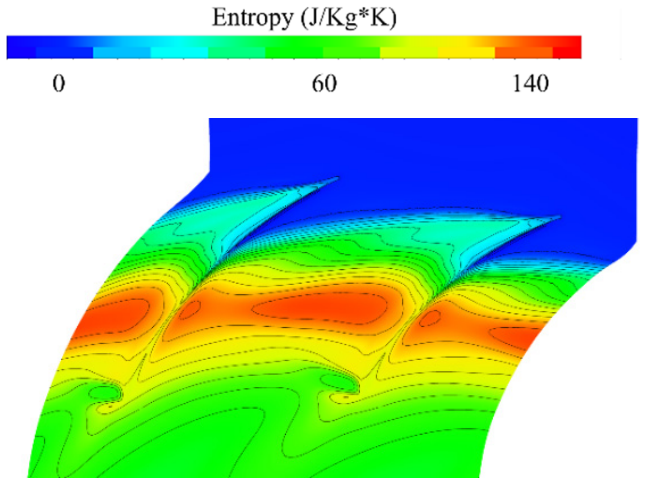

(a) prototype

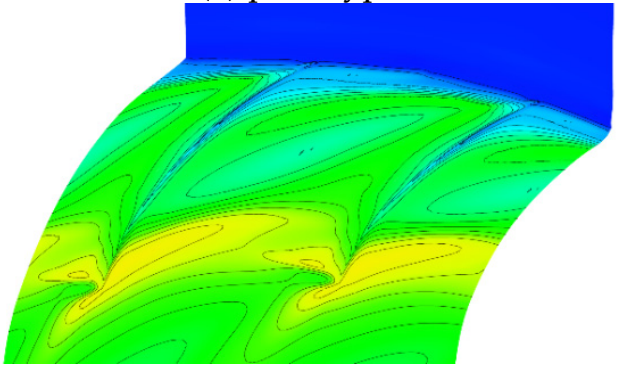

(b) optimal concave casing

Figure 12. The S1 surface entropy contour at $99 \%$ of the span.

Figure 13 shows the prototype EAT and the optimal concave casing tip leakage streamlines colored by the axial velocity, which passes the leading edge to $5 \%$ of the chord at the peak efficiency point. The secondary leakage of the prototype demonstrates that the fluid leakage at a large angle with the original suction surface passes the pressure surface of the adjacent blade at $30-50 \%$ of the blade after passing through the tip position and then mixes with the fluid that leaks near the suction surface of the adjacent blade and flows out of the blade channel. This secondary leakage is the main reason for the loss in the tip area of the prototype EAT. The deceleration-acceleration effect of the concave casing causes the clearance leakage fluid to decelerate at the leading edge; thus, the angle between the tip leakage vortex and the suction surface grows. In the middle chord region of the channel, the leakage flow accelerates again, and its ability to maintain the original direction of the movement becomes stronger, which significantly weakens the secondary leakage phenomenon, reduces the loss in the tip area, and improves the flow efficiency.

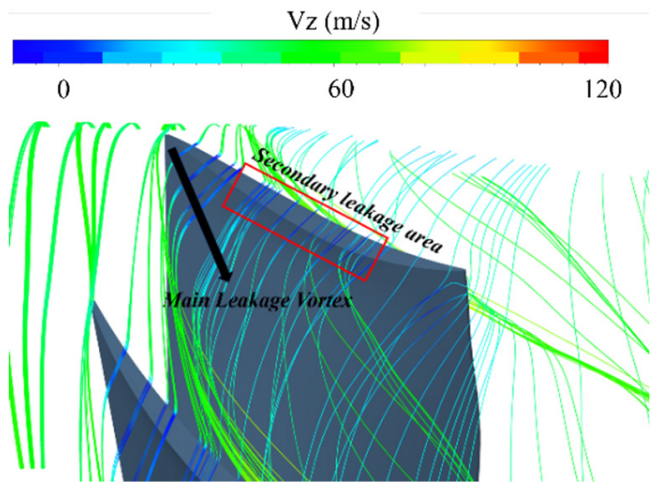

(a) prototype

Figure 13. Cont. 


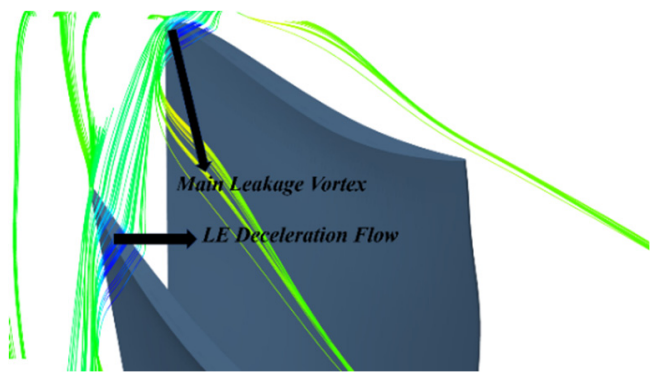

(b) optimal concave casing

Figure 13. Tip leakage flow streamlines.

\section{Endwall Optimization Design}

\subsection{Optimization Process and Result}

Comparing different abilities of a concave casing and concave hub to improve the EAT performance shows that because the concave casing improves the clearance leakage flow, the casing has a significant effect on the overall performance. In other words, the abovementioned discrete combination of the depth and position of the concavity improves the EAT rotor performance. Therefore, to realize the full potential of concave endwall modification, this paper proceeds with an EAT endwall optimization design. The details of the optimization progress are shown in Figure 14. The optimization was carried out on an CPU I7-6700K RAM 16 GB PC and took $83.7 \mathrm{~h}$ to complete.

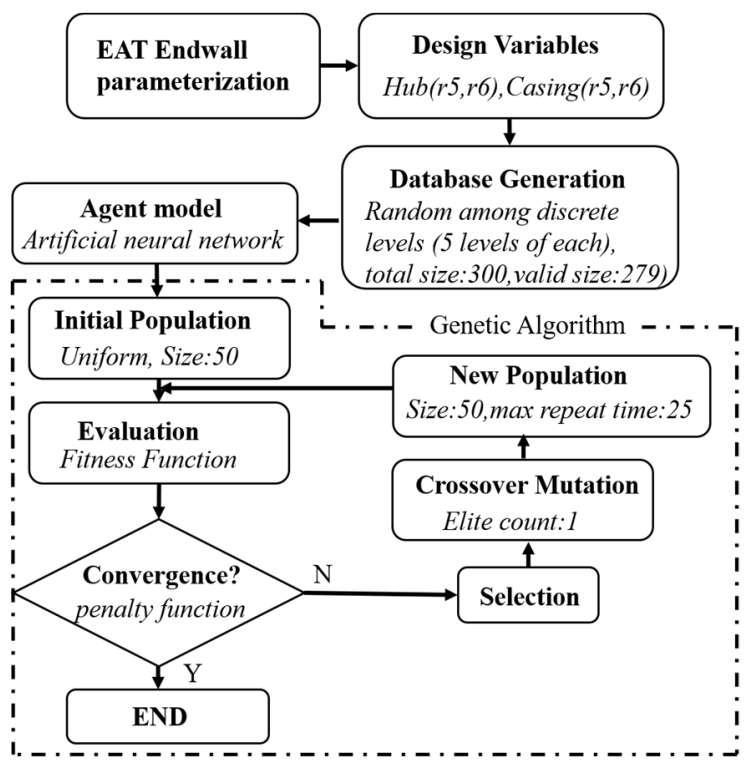

Figure 14. Optimization process.

Figure 15 shows comparisons between the characteristic lines of the prototype EAT and the optimized EAT and between their respective meridian surface profiles. The concave position of the optimized casing begins from the leading edge position and ends at $80 \%$ of the axial chord; the maximum concave position is at $64 \%$ of the axial chord, and the shape height is at $5.1 \%$ of $\mathrm{h}$. In contrast, the optimized hub is a convex hub. The convex position starts at $12 \%$ of the axial chord and ends at the trailing edge; the maximum convex position is located at $45 \%$ of the axial chord, and the shape height is $2.5 \%$ of $\mathrm{h}$. When applying the same boundary conditions as those employed for the prototype EAT design flow point, the total pressure ratio of the optimized EAT is increased by $0.67 \%$, the isentropic efficiency is 
increased by $1.5 \%$, and the mass flow rate is increased by $1.2 \%$. In terms of stability margin, the widely used equation employed for the general margin is shown in Equation (3).

$$
\operatorname{margin}=\left[\frac{\pi_{\text {stall }} \times m_{\eta \max }}{\pi_{\eta \max } \times m_{\text {stall }}}-1\right] \times 100 \%
$$

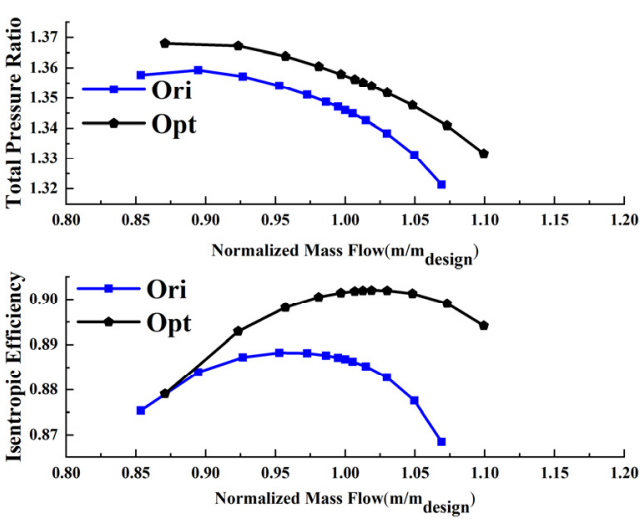

(a) Characteristic lines

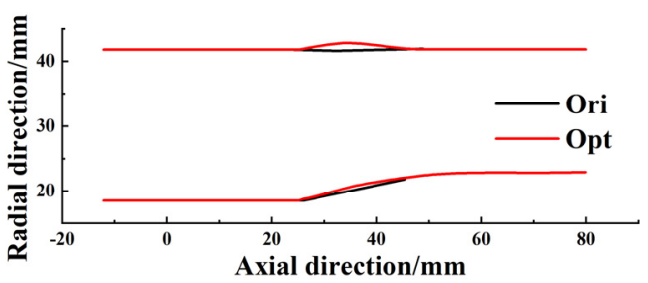

(b) Meridian endwall profiles

Figure 15. Comparisons between the prototype EAT and the optimized EAT.

The general margin of the prototype EAT is $13.6 \%$, while that of the optimized EAT is $19.96 \%$, which constitutes an increase of $6.36 \%$. In practical engineering applications, the working state of EAT is calibrated via flow. Therefore, this paper replaces the peak efficiency point parameter in Equation (3) with the design flow point parameter to obtain the design flow margin. Consequently, the design flow margin of the prototype EAT is $19.93 \%$, and the design flow margin of the optimized EAT is only $17.03 \%$. Compared with the prototype EAT, the design flow margin of the optimized EAT is reduced by $2.89 \%$, which indicates that the prototype EAT can achieve a stable working state with a lower flow rate. However, comparing the characteristic lines demonstrates that at the $0.629 \mathrm{~kg} / \mathrm{s}$ design flow point, the isentropic efficiency and pressure ratio of the optimized EAT are higher than those of the prototype EAT.

The concave hub is the result of the summary based on the previous study (which was conducted by other researchers). The author assumed that the concave hub can also make a positive effect on the performance of EAT, and it turned out exactly as we expected. The convex hub is the optimization result (which was generated by the algorithm), and the author tended to think that the combination effect of the convex hub and the concave casing resulted in the improved performance. The major reason is still the casing optimization.

\subsection{Analysis of the Optimization Results}

To compare the differences in clearance flow between the optimized EAT and the prototype EAT at the design flow point and the near-stall point, this paper defines two dimensionless axial momentum coefficients, as shown in Equations (4) and (5).

$$
\begin{gathered}
\mu=\int_{r_{\text {tip }}}^{r_{\text {casing }}} \frac{\rho V_{n} V_{z}}{\left(\rho V_{n} V_{z}\right)_{\text {ref }}} d \bar{r} \\
C_{\mu}=\iint \frac{\rho V_{n} V_{z}}{\left(\rho V_{n} V_{z}\right)_{\text {ref }}} d A
\end{gathered}
$$

$V_{n}$ is the velocity component vertical with respect to the clearance, $V_{z}$ is the axial velocity, $\bar{r}$ is the uniform clearance height, $A$ is the clearance area, $\mu$ is the radial integral value, and $C_{\mu}$ is the surface integral value [15]. Due to the small curvature of the tip element camber at the leading edge and trailing edge, part of the velocity distribution is oriented in the 
same direction as the mainstream velocity at the leading edge and trailing edge where $\mu>$ 0 . Therefore, to distinguish the different directions between the tip leakage flow and the main flow, $C_{\mu}$ will be integrated according to the sign.

Figure 16 shows that at the design flow point, the maximum leakage flow strength of the prototype EAT is located at $35 \%$ of the chord, and secondary leakage occurs at $60-80 \%$ of the chord length. In contrast, the optimized EAT decelerates the flow in the leading edge region of the blade tip. Additionally, compared with the prototype EAT, the leakage flow strength of the optimized EAT reaches its peak at $25 \%$ of the chord length, and the maximum strength is higher than that of the prototype EAT, but due to the concave endwall contraction effect, the leakage flow accelerates out of the channel. At the near-stall point, due to the increased aerodynamic load, the leakage peak position of the prototype EAT moves forward to $10 \%$ of the chord length, and the extent of secondary leakage is also expanded to $40-75 \%$ of the chord, causing greater loss. Compared with the prototype EAT, as a result of the leading edge deceleration effect of the optimized EAT, the peak leakage flow is still greater than that of the prototype EAT, but the axial forward movement of the leakage peak is lower than that of the prototype EAT. This indicates that the influence region of high-strength leakage flow in the optimized EAT is smaller than that of the prototype EAT under variable working conditions. In addition, there is no obvious secondary leakage in the optimized EAT, indicating that with an increase in aerodynamic load, the acceleration effect of the concave endwall in the second half is also enhanced. At this time, the mainstream and leakage flows are simultaneously accelerated by meridian contraction, which suppresses the occurrence of secondary leakage.

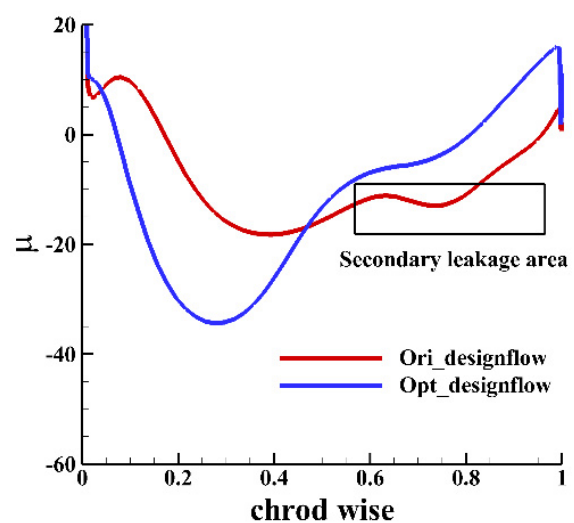

(a) Design flow point

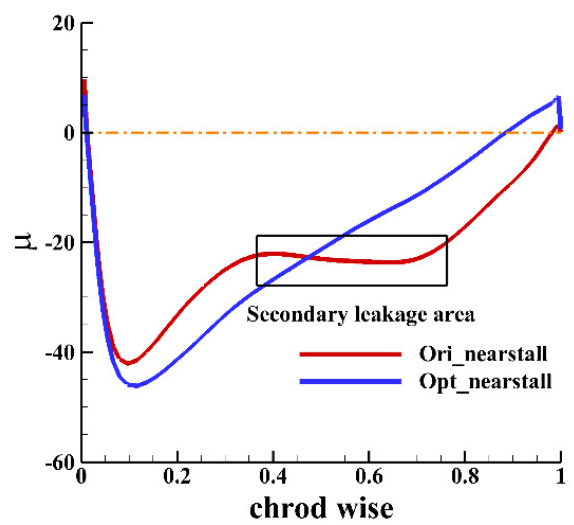

(b) Near-stall point

Figure 16. Distribution of the tip clearance dimensionless axial momentum coefficient.

On the basis of the area integration results for the dimensionless axial momentum coefficient plotted in Figure 17, the optimized EAT has higher leakage flow strength than does the prototype EAT at the design flow point, At the same time, the leakage part with the same direction as the mainstream flow in the optimized EAT is also stronger than that in the prototype EAT. According to Figure 16, due to the deceleration-acceleration effect of the concave endwall on the flow, part of the flow oriented in the same direction as the main flow is concentrated between $80 \%$ of the chord length and the trailing edge; this part of the flow enhances the low-energy flow that accumulates at the suction surface tip and the trailing edge corner. At the near-stall point, the tip leakage flow strength of the optimized EAT is weaker than that of the prototype EAT, and some flow remains with a positive axial momentum at the trailing edge, which enhances the low-energy flow. 


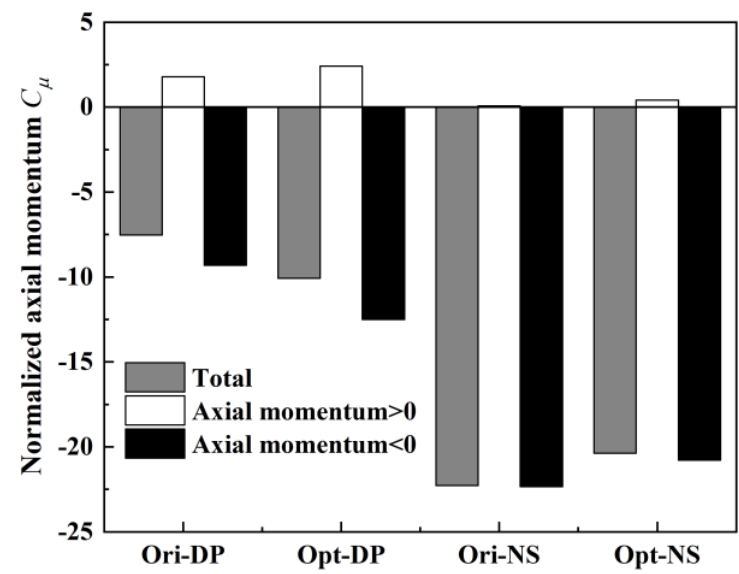

Figure 17. The area integral of the nondimensional axial momentum coefficient in tip clearance.

To compare the different flow structures between the prototype EAT and the optimized EAT under approximately the same flow blockage state, the flow coefficient $\Psi$ is defined in Equation (6), where $v_{x}$ is the mass average axial velocity from $98 \%$ of the span to the casing and $U_{\text {tip }}$ is the tangent speed of the blade tip.

$$
\Psi=v_{x} / U_{t i p}
$$

Figure 18 compares the inlet flow angles at the prototype EAT peak efficiency point ( $\Psi=0.478)$, the optimized EAT peak efficiency point $(\Psi=0.517)$, and the optimized EAT point $(\Psi=0.478)$. The incoming flow angle at the optimized EAT point is lower than that the prototype EAT peak efficiency point in the full span range, which weakens the leading edge separation phenomenon caused by an increase in the incoming flow angle.

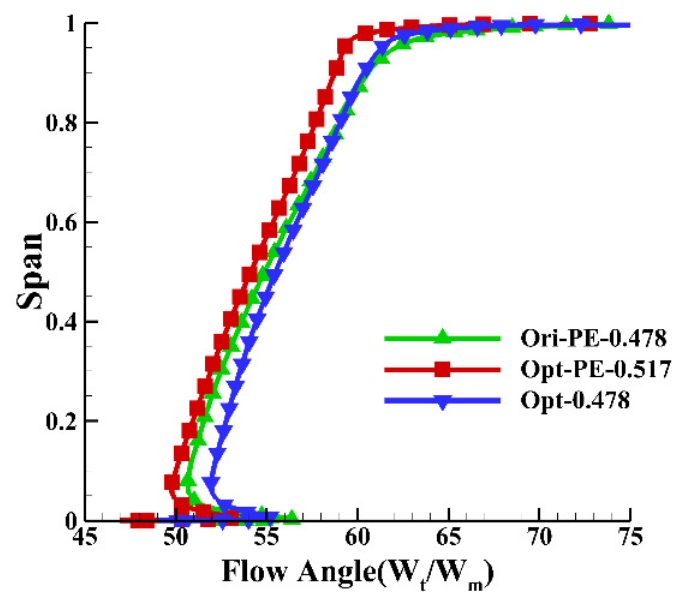

Figure 18. Comparison of the inlet angle at different working points.

The separation at the leading edge caused by the increase in the inlet angle in a single rotor occurs due to two main mechanisms. The first mechanism is the separation of flow at the suction surface blade tip corner area. This separation acts on the entire blade channel, causing the incoming flow of adjacent blades to be deviated, thereby increasing the inlet flow angle and causing the flow at the leading edge of the tip to separate. The second mechanism is an increase in the inlet angle caused by the tip leakage vortex. These two mechanisms can inhibit each other under a certain clearance height, causing the inlet angle to decrease and weakening the flow caused by the separation of the leading edge. In the optimized EAT ( $\Psi=0.478)$, at $90-100 \%$ of the span, the inlet angle is also lower than that in the prototype EAT, which broadens the stable working range of the EAT rotor and improves the general margin. The above discussion suggests that the high blade span part 
of the EAT allows more airflow to pass, and the flow improvement of the high blade span strongly enhances the overall performance.

The separation at the leading edge caused by the increase in the inlet angle in a single rotor occurs due to two main mechanisms. The first mechanism is the separation of flow at the suction surface blade tip corner area. This separation acts on the entire blade channel, causing the incoming flow of adjacent blades to deflect, thereby increasing the inlet flow angle and causing the flow at the leading edge of the tip to separate. The second mechanism is an increase in the inlet angle caused by the tip leakage vortex. These two mechanisms can inhibit each other under a certain clearance height, causing the inlet angle to decrease and weakening the flow caused by the separation of the leading edge. In the optimized EAT ( $\Psi=0.478$ ), at $90-100 \%$ of the span, the inlet angle is also lower than that in the prototype EAT, which broadens the stable working range of the EAT rotor and improves the general margin. The above discussion suggests that the high blade span part of the EAT allows more airflow to pass, and the flow improvement of the high blade span strongly enhances the overall performance.

\section{Influences of Different Clearance Sizes}

In the above discussion, the clearance size of the optimized EAT is consistent with that of the prototype EAT, which is $0.34 \mathrm{~mm}$ ( $1 \%$ blade tip chord length, 10 clearance). Considering that the clearance size will change due to machining errors and under different operating conditions, to compare the performance between the prototype EAT and the optimized EAT under different clearance sizes. To select the optimal clearance, the prototype EAT and the optimized EAT characteristic lines are simulated under design clearance sizes of $50 \%, 150 \%$, and $200 \%$ (05 clearance, 15 clearance, and 20 clearance, respectively). Moreover, to explore the effect of tip leakage flow on the performance, this paper also simulates the characteristic lines of the prototype EAT and the optimized EAT with a $0 \%$ clearance size (00 clearance). Although rotor tip sealing technology cannot be applied to mass-produced EATs, for this research, the characteristic line at $0 \%$ clearance still has value.

\subsection{Comparison of Different Clearance Size Characteristic Lines}

Figure 19a,b show a comparison between the characteristic lines of the prototype EAT and the optimized EAT under different clearance sizes. For the prototype EAT, the peak isentropic efficiency first increases and then decreases with an increasing clearance size. The peak isentropic efficiency is $91.27 \%$, and the corresponding clearance size is $50 \%$ of the design clearance. The change in the total pressure ratio follows the same trend as the change in isentropic efficiency, and the peak total pressure ratio is 1.389. The characteristic line of the prototype EAT indicates that a certain blade tip clearance size improves the performance. For the prototype EAT, considering both the total pressure ratio and the isentropic efficiency, the optimal clearance is $50 \%$ of the design clearance.

For the optimized EAT, the peak isentropic efficiency decreases with an increasing clearance size, the peak isentropic efficiency is $93.14 \%$, the corresponding gap size is 0 , the peak total pressure ratio decreases with an increasing gap size, and the peak total pressure ratio is 1.397. Both sets of characteristic lines reveals that from the perspective of the pressure ratio and efficiency, the clearance size has different influences on the prototype EAT and the optimized EAT. In the following, this phenomenon will be analyzed from two perspectives: the spanwise distribution of the average outlet total pressure ratio and the relative total pressure distribution of the S1 stream surface at $99 \%$ of the span. 


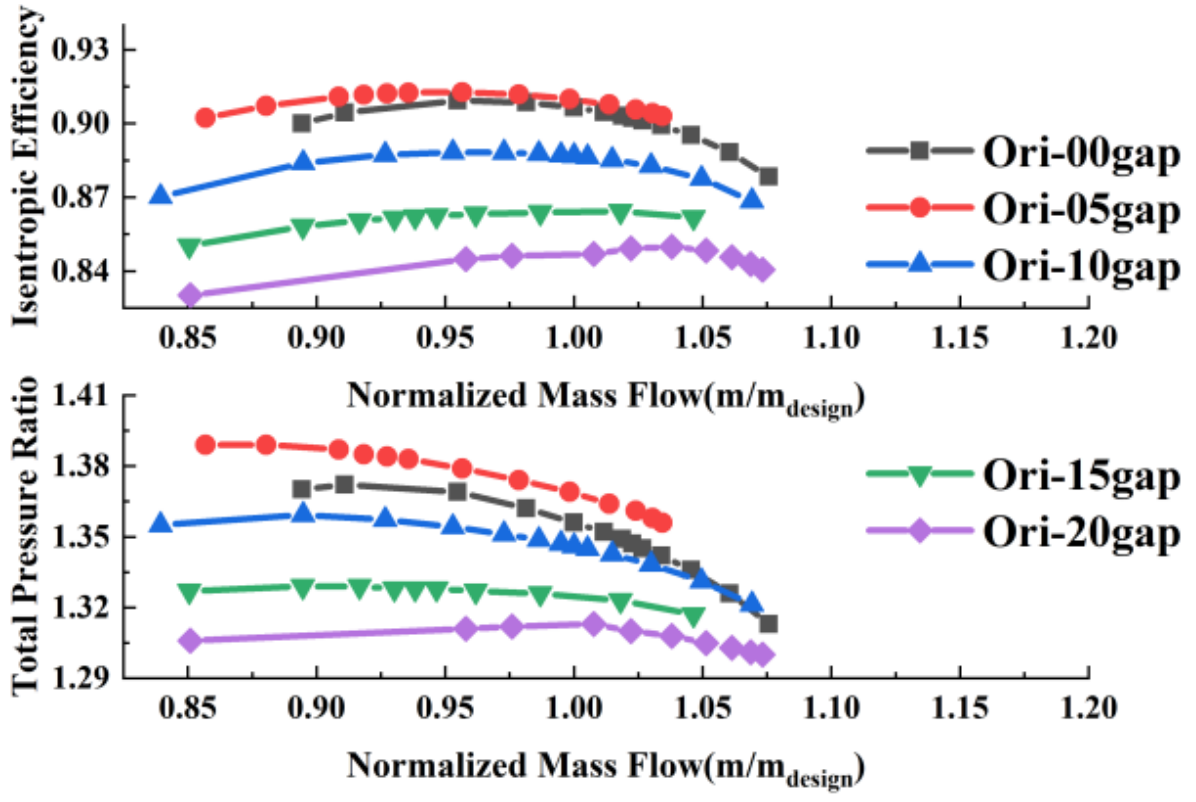

(a) Prototype EAT
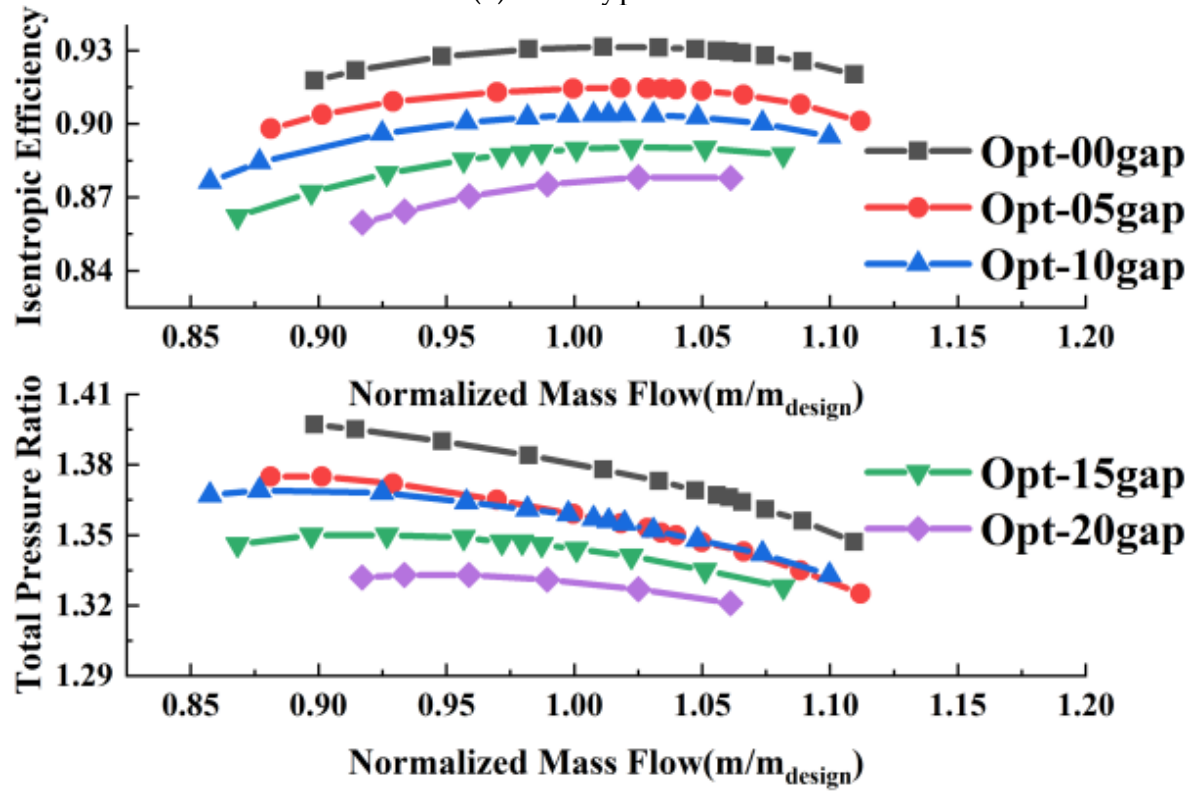

(b) Optimized EAT

Figure 19. Comparison of characteristic lines under different clearance sizes.

\subsection{Analysis of Performance Variation}

According to the previous description of the prototype EAT, a certain clearance width increases the total pressure ratio and efficiency. The main reason for the change in this mechanism in the optimized EAT is that the concave casing makes the main flow structure of the blade tip first decelerate and subsequently accelerate. In the prototype EAT, there is not only mutual inhibition of corner separation and tip leakage vortex but also a deceleration-acceleration effect caused by the concave casing. These two effects cause the optimized EAT's peak total pressure ratio and peak isentropic efficiency to gradually decrease with an increasing clearance size. Figure 20a shows the spanwise distribution of the outlet total pressure ratio at the peak efficiency point under the $0 \%$ clearance size of the prototype EAT. Due to the $0 \%$ clearance state, there is no tip leakage flow. The total pressure ratio distribution can be divided into three main sections: $A, B$, and $C$. Section $C$ contains the pressure ratio change caused by the uplift of flow in the hub area due to the centrifugal force, Coriolis force, and hub contraction. Section B is the middle section of the blade where 
the flow separation is minor; the main factor affecting the change in the pressure ratio here is the blade tangential velocity, which increases with the radius. Section A involves the flow close to the casing region; in the $0 \%$ gap state, the main factor for the change in this section is the corner separation of the suction surface trailing edge. Figure 20b shows the spanwise distribution of the outlet total pressure ratio at the peak efficiency point for the prototype EAT under $0 \%$ clearance, $50 \%$ clearance, and $100 \%$ clearance. Below $60 \%$ of the span, the three total pressure ratio curves basically coincide. For section A under $0 \%$ clearance, the pressure ratio at the $50 \%$ clearance size is higher than that at the $0 \%$ clearance size. This is mainly due to the increase in tip leakage flow caused by the gradually increasing tip clearance, which inhibits suction surface corner separation. Compared with the positions under $0 \%$ clearance and $100 \%$ clearance, section B under $50 \%$ clearance is closer to the casing position. These findings show that in the prototype EAT, for the pressure ratio (efficiency), there is an optimal gap; hence, the tip leakage flow and the suction surface corner separation can reach a balanced point, thereby achieving the maximum pressure ratio (efficiency).

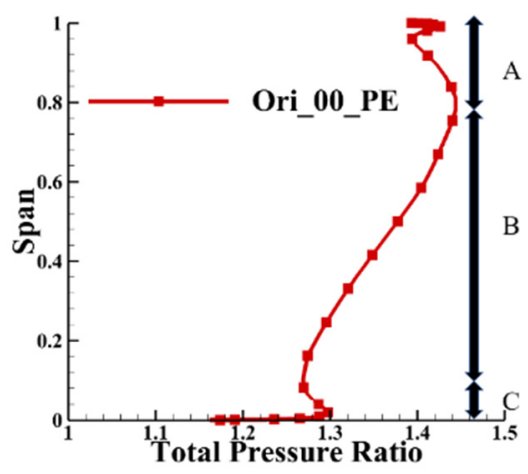

(a) Prototype $0 \%$ clearance

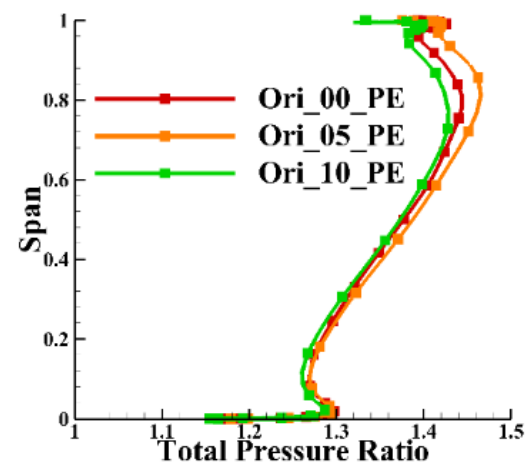

(b) Prototype $0 \%, 50 \%$, and $100 \%$ clearance

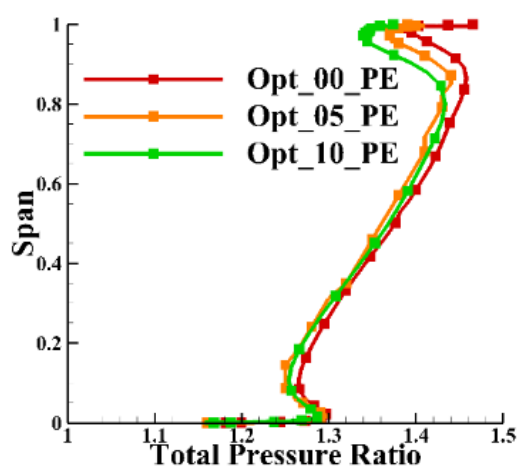

(c) Optimized $0 \%, 50 \%$, and $100 \%$ clearance

Figure 20. Spanwise distributions of the outlet total pressure ratio.

Figure 20c shows the spanwise distribution of the outlet total pressure ratio at the peak efficiency point for the optimized EAT under $0 \%, 50 \%$, and $100 \%$ clearance sizes. For the pressure ratio changes under $0 \%$ clearance, 50\% clearance, and 100\% clearance, Section $\mathrm{A}$ is narrower than that in the prototype EAT, which means that the region influenced by the tip leakage flow and suction surface corner separation is smaller in the optimized EAT. With an increase in clearance size, the increase in tip leakage flow begins to suppress corner separation, but the same pressure ratio increases, unlike in the prototype EAT. The reason for this phenomenon is that the starting position of the tip leakage flow is near the leading edge, while suction surface corner separation occurs near the trailing edge. The tip leakage flow is decelerated and then accelerated by the concave endwall, whereas the suction surface corner separation is only subject to acceleration, so the separation has a stronger ability to maintain the original movement.

Figure 21 shows the axial velocities of the prototype EAT and the optimized EAT at $99 \%$ of the flow channel height under $0 \%, 50 \%$, and $100 \%$ clearance. Compared to the prototype EAT, the low-speed area caused by the corner separation in the optimized EAT is still obvious under the $50 \%$ gap state. Under $100 \%$ clearance, the different lowvelocity areas caused by tip leakage flow and suction surface corner separation can still be distinguished. 


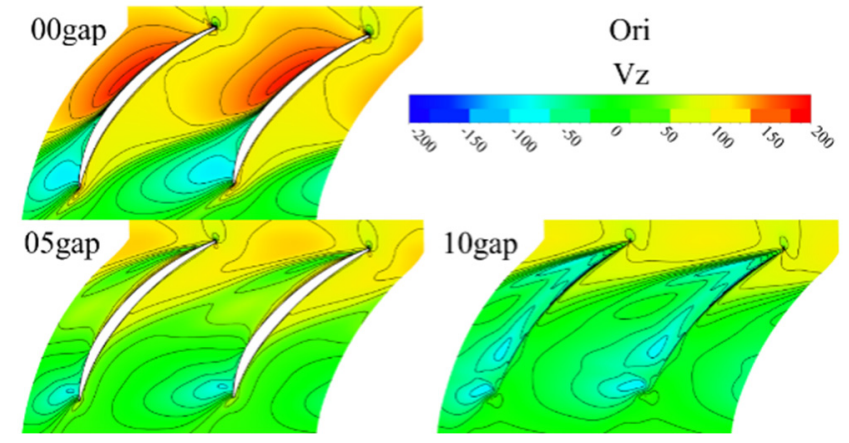

(a) Prototype EAT

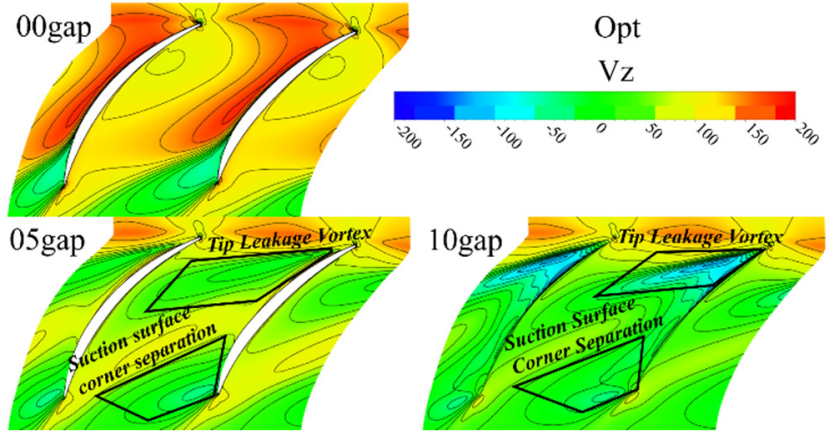

(b) Optimized EAT

Figure 21. The S1 surface axial velocity at $99 \%$ of the span.

Similar to the case for all axial compressors, a stable margin is important to ensure that the EAT can operate normally within a periodically changing backpressure environment. Figure 22 shows the variation in the flow coefficient difference $\Delta \Psi$ and the general margin with the clearance size between the prototype EAT and the optimized EAT at the peak efficiency point and the near-stall point. For both the prototype EAT and the optimized EAT, the trend of $\Delta \Psi$ with the tip clearance is the same as that of the general margin. The general margin of the prototype EAT increases with increasing clearance size, while the general margin of the optimized EAT first increases and then decreases with an increasing clearance size. The margin reaches the maximum value at $100 \%$ of the design clearance size. Moreover, under $0 \%, 50 \%$, and $100 \%$ clearance, the general margin of the optimized EAT is higher than that of the prototype EAT. For the prototype EAT, as the clearance size gradually increases, the leakage flow has a greater impact on the flow in the full blade span. The characteristic lines show that the margin of the prototype EAT increases at the expense of a decrease in the pressure-rise capacity. Additionally, under $200 \%$ clearance, the peak efficiency point pressure ratio is reduced by $3.4 \%$ compared with that under $100 \%$ clearance. For the optimized EAT, as the clearance size increases, the pressure-rise capacity decreases less than that of the prototype EAT. Under the $200 \%$ design clearance state, the peak efficiency point pressure ratio drops by only $2 \%$ compared with that under the $100 \%$ design clearance state. Furthermore, the main factor for the increase in the leading edge inlet angle changes from suction surface corner separation to tip leakage flow. Therefore, on the basis of the general margin, there is an optimal clearance size. In the scheme studied in this paper, the optimal clearance of the optimized EAT is $100 \%$ of the design clearance. It is worth noting that in the optimized EAT, the change trend of the general margin is the same as that of the spike-stall rotor studied in Reference [15]. Hence, the stall form of the optimized EAT may also be spike-stall. However, the specific form of stall still needs to be simulated with an unsteady simulation.

As we can see from comparing results between the prototype and the optimization in different size clearance, it seems that the prototype with a narrower clearance has a better performance than the optimized EAT. However, considering that once put into practice, a small increase in manufacturing accuracy may result in a high cost increase. Compared with changing the already narrow default tip clearance $(0.34 \mathrm{~mm})$, the axisymmetric endwall modification has an obvious performance effect and a limited cost increase. Therefore, instead of using the clearance reduction scheme of the prototype EAT, an axisymmetric endwall modification with the same clearance dimensions as the prototype is adopted. 


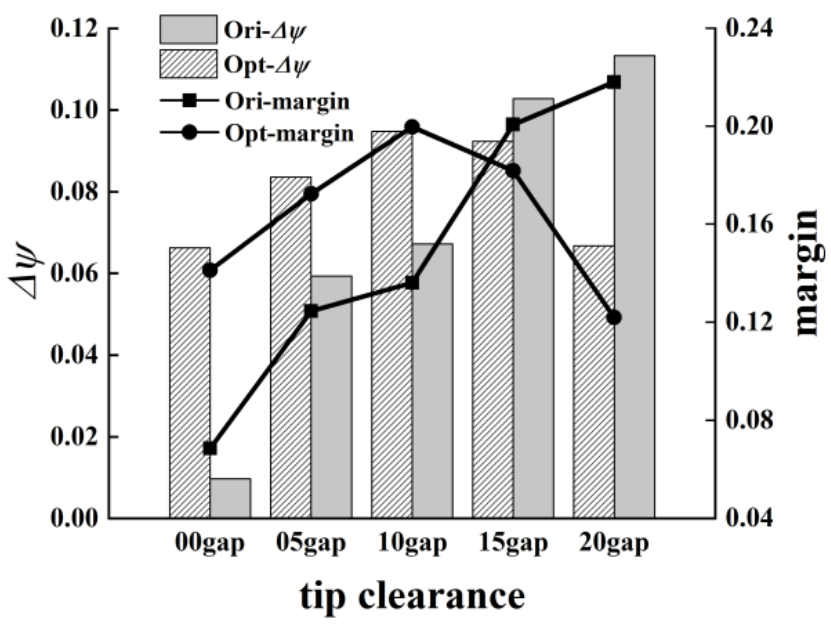

Figure 22. Differences in the flow coefficient and general margin change with the clearance size.

\section{Conclusions and Future Work}

To further improve the performance of the EAT, the effect of axisymmetric endwalls were investigated via numerical simulation. Discrete schemes were first studied; then, the optimized endwall was obtained using a backpropagation artificial neural network and a genetic algorithm. The effects of different clearance sizes on the prototype EAT and the optimized EAT were discussed in detail. The main conclusions are as follows:

For concave hubs, the deceleration-acceleration effect of the endwall on the fluid reduces the load at the root of the blade and the lateral pressure gradient of the hub, thus improving the flow state. In the modified optimal concave hub, the efficiency at the peak efficiency point increases by $0.24 \%$. For the concave casing, the deceleration effect caused by the expansion part of the endwall shifts the start position of the tip leakage vortex forward to variable working points. Moreover, the contraction part of the endwall increases the axial velocity. The ability of the tip leakage vortex to maintain the original direction is enhanced, decreasing the secondary leakage flow loss on the overall performance. In the optimal concave casing modification, the efficiency of the peak efficiency point is increased by $0.71 \%$. In general, the concave casing had a more obvious effect than did the concave hub on the EAT performance.

Optimization design was carried out by using a backpropagation artificial neural network and a genetic algorithm, and a convex hub and full-chord concave casing were obtained. The convex hub decelerated the flow in the blade root region and weakened the profile loss, while the full-chord concave casing deceleration-acceleration effect decreased the loss which caused by the tip leakage flow. At the design mass flow point, the isentropic efficiency and the pressure ratio both increased and the peak efficiency increased by $2.2 \%$.

Under different clearance sizes, for the prototype EAT, the tip leakage flow obviously weakened the suction surface corner separation at $50 \%$ of the design clearance. For the optimized EAT, the low-velocity region caused by suction surface corner separation could still be distinguished at $50 \%$ and $100 \%$ of the design clearance. The concave casing made the tip leakage flow decelerate first and then accelerate, thereby accelerating the suction surface corner separation; thus, the change trends of the efficiency and general margin varied with the clearance size and were different between the prototype EAT and the optimized EAT.

Axisymmetric endwall technology has shown great potential in the EAT performance improvement, mainly because the tip region flow significantly influenced the global performance. Future work will combine the axisymmetric endwall technology, the bend and sweep blade technology, and tip winglet technology to further improve the EAT performance, research the composite mechanism of these three technologies, and obtain the optimal scheme of the EAT geometry from the view of aerodynamics design. 
Author Contributions: Conceptualization, J.Z. and H.T.; methodology, H.T.; software, H.T.; validation, J.Z. and W.W.; formal analysis, H.T.; investigation, H.T.; resources, H.T.; data curation, H.T.; writing-original draft preparation, H.T.; writing—review and editing, W.W.; visualization, H.T.; supervision, H.T.; project administration, J.Z.; funding acquisition, J.Z. and W.W. All authors have read and agreed to the published version of the manuscript.

Funding: The research is funded by National Nature Science Foundation of China (Grant No. 51906134; 51436002).

Institutional Review Board Statement: Not applicable for studies not involving humans or animals.

Informed Consent Statement: Not applicable.

Data Availability Statement: Data sharing not applicable.

Acknowledgments: The authors would like to thank Chen D.Y. of China Aerodynamics Research and Development Center for his precious and constant support as well as for his invaluable suggestions during the simulation activities.

Conflicts of Interest: The authors declare no conflict of interest.

\section{References}

1. Burke, R.D. A numerical study of the benefits of electrically assisted boosting systems. J. Eng. Gas. Turbines Power 2016, 138. [CrossRef]

2. Rothgang, S.; Pachmann, M.; Nigrin, S.; von Scheven, M. The Electric Supercharger Challenge, Conception and Implementation. MTZ Worldw. 2015, 76, 4-9. [CrossRef]

3. Zhang, Y. Analysis of the development status and trend of fuel cell vehicle air compressor. I.C.E Parts 2019, 1, 201-202. [CrossRef]

4. Zhang, K.S.; Wang, G.H.; Li, G.X. The Development of Electrically Assisted Turbocharger. I.C.E Powerpalant. 2008, 2, 31-35.

5. Hoeger, M.; Cardamone, P.; Fottner, L. Influence of Endwall Contouring on the Transonic Flow in a Compressor Blade, Volume 5: Turbo. Expo. 2002, Parts A and B, Amsterdam. In Proceedings of the ASME Turbo Expo 2002: Power for Land, Sea, and Air, Amsterdam, The Netherlands, 3-6 June 2002; pp. 759-768. [CrossRef]

6. Kröger, G.; Cornelius, C.; Nicke, E. Rotor Casing Contouring in High Pressure Stages of Heavy Duty Gas Turbine Compressors With Large Tip Clearance Heights, Volume 7: Turbomachinery, Parts A and B. In Proceedings of the ASME Turbo Expo 2009: Power for Land, Sea, and Air, Orlando, FL, USA, 8-12 June 2009; pp. 215-225. [CrossRef]

7. Kröger, G.; Voß, C.; Nicke, E.; Cornelius, C. Theory and Application of Axisymmetric Endwall Contouring for Compressors, Volume 7: Turbomachinery, Parts A, B, and C. In Proceedings of the ASME 2011 Turbo Expo: Turbine Technical Conference and Exposition, Vancouver, BC, Canada, 6-10 June 2011; pp. 125-137. [CrossRef]

8. Xu, Q.Y.; Hou, A.P.; Li, S.B.; Zhou, S. Influences of Hub Design on Rotor Performance in Transonic Compressor. J. Eng. Thermophys. 2009, 30, 761-764.

9. Yang, C.; Li, Q.S.; Yuan, W.; Zhou, S. Effect of Hub-Shape Contouring on The Corner Blockage of Compressor Stator Cascade. J. Aero Power 2009, 24, 2333-2337.

10. Zhang, L.X.; Zhou, X.; Wu, F.; Du, X.; Wang, S.T.; Wang, Z.Q. Application Study of Concave Axisymmetric Endwall Molding in a Compressor Cascade with High Stagger Angle. J. Propulsion. Technol. 2016, 37, 1870-1876. [CrossRef]

11. Sun, S.J.; Chen, S.W.; Liu, W.; Wang, S.T. Impact of Shroud Contouring on Performance of Low-Reaction Transonic Highly Loaded Rotor. J. Propulsion. Technol. 2018, 39, 2710-2717. [CrossRef]

12. Liu, X.F.; Liu, S.W.; Yang, X.H. Numerical Calculation of Hub-shape Contouring on a High Bypass Ratio Engine Fan. Aeroengine 2018, 44, 26-33. [CrossRef]

13. Gao, Y. Investigation of Transonic Compressor Rotor for Three-dimensional Aerodynamic Design and Experiment. Ph.D. Thesis, Dalian Maritime University, Dalian, China, 2017.

14. Wu, W.Y. Investigation of High Subsonic Compressor Cascade Tip Clearance Control Using Blade Tip Winglet. Ph.D. Thesis, Dalian Maritime University, Dalian, China, 2018.

15. Hewkin-Smith, M.; Pullan, G.; Grimshaw, S.D.; Greitzer, E.M.; Spakovszky, Z.S. The Role of Tip Leakage Flow in Spike-Type Rotating Stall Inception, Volume 2D: Turbomachinery. In Proceedings of the ASME Turbo Expo 2017: Turbomachinery Technical Conference and Exposition, Charlotte, NC, USA, 26-30 June 2017. [CrossRef] 